\title{
A Novel Mass Spectrometry Cluster for High-Throughput Quantitative Proteomics
}

\author{
Magnus Palmblad, Yuri E. M. van der Burgt, Ekaterina Mostovenko, \\ Hans Dalebout, and André M. Deelder \\ Biomolecular Mass Spectrometry Unit, Department of Parasitology, Leiden University Medical Center, \\ Leiden, The Netherlands
}

\begin{abstract}
We have developed and implemented a novel mass spectrometry (MS) platform combining the advantages of high mass accuracy and resolving power of Fourier transform ion cyclotron resonance (FTICR) with the economy and speed of multiple ion traps for tandem mass spectrometry. The instruments are integrated using novel algorithms and software and work in concert as one system. Using chromatographic time compression, a single expensive FTICR mass spectrometer can match the throughput of multiple relatively inexpensive ion trap instruments. Liquid chromatography (LC)-mass spectrometry data from the two types of spectrometers are aligned and combined to hybrid datasets, from which peptides are identified using accurate mass from the FTICR data and tandem mass spectra from the ion trap data. In addition, the high resolving power and dynamic range of a 12 tesla FTICR also allows precise label-free quantitation. Using two ion traps in parallel with one LC allows simultaneous MS/MS experiments and optimal application of collision induced dissociation and electrontransfer dissociation throughout the chromatographic separation for increased proteome coverage, characterization of post-translational modifications and/or simultaneous measurement in positive and negative ionization mode. An FTICR-ion trap cluster can achieve similar performance and sample throughput as multiple hybrid ion trap-FTICR instruments, but at a lower cost. We here describe the first such FTICR-ion trap cluster, its performance and the idea of chromatographic compression. (J Am Soc Mass Spectrom 2010, 21, 1002-1011) (c) 2010 American Society for Mass Spectrometry
\end{abstract}

$\mathrm{I}$

n recent years, mass spectrometry (MS) has become a popular method for identification and quantitation of proteins and metabolites in complex biological matrices. The reasons for this are at least 2-fold: mass spectrometry can separate a very large number of chemical species of different mass in a complex sample and, secondly, unknown peptides or proteins can be routinely and automatically identified by data-dependent tandem mass spectrometry (MS/MS). The resolution or peak capacity is increased further by coupling the mass spectrometer to a liquid chromatography (LC) system. Analytical challenges from the rapidly expanding field of proteomics have pushed the development of mass spectrometers in general and led to further optimization of systems for peptide and protein analysis.

The workhorses in MS-based proteomics are ion traps [1]. These are perfectly suited for on-line coupling to $\mathrm{LC}$ via electrospray ionization (ESI) and are capable of analyzing complex peptide mixtures by rapid MS/ MS. Although ion traps are sensitive and versatile, they have relatively low resolving power $\left(<10^{4}\right)$ and mass measurement accuracy ( $\sim 100 \mathrm{ppm})$. In modern mass

Address reprint requests to Dr. Magnus Palmblad, Department of Parasitology, Leiden University Medical Center, P.O. Box 9600, 2300 RC Leiden, The Netherlands. E-mail: n.m.palmblad@lumc.nl spectrometers, quadrupoles or ion traps are often used in hybrid configuration with a second mass analyzer, such as time-of-flight [2] (TOF), Orbitrap [3], or FTICR $[4,5]$, within a single vacuum system. In these hybrid instruments, the ion trap provides rapid, sensitive MS/ MS, or at least precursor ion selection, and the second analyzer accurate mass and high resolving power. The hybrid linear ion trap-Fourier transform ion cyclotron resonance (FTICR) mass spectrometer [4] can be considered to represent the current state-of-the-art in commercially available mass spectrometry instrumentation.

An alternative paradigm to combining two mass analyzers in one physical instrument is to merge data from two different instruments analyzing the same sample. Several analytical strategies have been developed based on this general idea, notably the accurate mass and time tag (AMT) approach of Smith et al. [6], wherein MS/MS data from ion traps are used to validate peptide identifications based on accurate mass and to train a predictor of chromatographic retention times [7, 8]. Accurate MS and ion trap MS/MS data can also be directly combined using chromatographic alignment $[9,10]$.

All published hybrid instrument designs and previously published data fusion schemes are inefficient, at least timewise, in their use of the accurate mass detector. 
This is because the latter invariably has much higher resolving power than the ion trap used for MS/MS and thus is able to detect most species selected for MS/MS on a considerably shorter chromatographic time scale.

Given the difference in cost and size of ion traps (small and relatively inexpensive) and Orbitrap or FTICR mass spectrometers (larger and more expensive), it also makes economic sense to combine multiple ion traps with a single FTICR mass spectrometer. We have therefore designed and implemented an integrated FTICR-ion trap cluster, a system of mass spectrometers that work together as a single entity to analyze one sample stream using differential chromatographic gradients for LC-MS and LC-MS/MS. The use of a dedicated instrument platform is novel, but it is the concept and use of different chromatographic time scales that is important, rather than the exact configuration or number of ion traps. We will therefore describe the performance and consequence of chromatographic compression in detail and exemplify this approach with an application in a quantitative proteomics study.

\section{Methods}

\section{Test Samples}

For evaluation of the FTICR-ion trap cluster, we repeated the classic glucose/lactose diauxie experiment by Jacob and Monod [11]. E. coli K12 strain MG1655 was acquired from ATCC and cultured in 1 L MOPS minimal medium with $0.5 \mathrm{~g} / \mathrm{L}$ glucose and $1.5 \mathrm{~g} / \mathrm{L}$ lactose in a 3 L fermentor (Applikon Biotechnology, Schiedam, The Netherlands), duplicating as closely as possible the recent glucose/lactose diauxie gene expression study by Traxler et al. [12]. The culture was monitored by spectrophotometric OD600 measurement, glucose concentration measured using a glucose oxidase assay kit (Sigma-Aldrich Chemie B.V., Zwijndrecht, The Netherlands) and lactose concentration followed using a galactosidase/lactose kit (BioVision, Mountain View, CA, USA). Three replicate cultures were sampled at approximately $-100,-50,-10,0,10,20,30,40,50$, and $60 \mathrm{~min}$ relative to the diauxic shift. Proteins were extracted using the Novagen "BugBuster" kit (Merck $\mathrm{KGaA}$, Darmstadt, Germany), following the manufacturer's recommended protocol with $5 \mathrm{~mL}$ of lysis buffer per gram of wet cell weight.

For identification, $25 \mu \mathrm{g}$ protein from two time points, one before and one after the diauxic shift, was fractionated using SDS-PAGE (NuPAGE 8\%-12\%; Invitrogen, Carlsbad, CA, USA) by cutting the gel lane into 26 two-mm bands, reduced (10 $\mathrm{mM} \mathrm{DTT}, 56{ }^{\circ} \mathrm{C}, 45$ $\mathrm{min}$ ), alkylated (iodoacetamide, room temperature, $1 \mathrm{~h}$ in dark) and digested in-gel using trypsin (sequencing grade; Promega, Madison, WI, USA). For quantitative measurement, $250 \mu \mathrm{g}$ of protein from each individual sample was digested as above but in solution and $2 \mu \mathrm{g}$ of each digest injected on column.
To compare the sensitivity and quantitative precision, bovine serum albumin (BSA) was spiked into an E. coli protein extract from a post-diauxie time point at BSA-to-E. coli ratios of $0,0.01 \%, 0.1 \%, 1 \%$, and $10 \%$. The digestion protocol was the same as for the gel slices except for the omission of the destaining and washing steps. The spiked samples were analyzed in an iterated sequence from low to high concentration BSA, with a blank after the highest concentration.

\section{FTICR-Ion Trap Cluster}

All LC systems in the FTICR-ion trap cluster are parallel, splitless NanoLC-Ultra 2D plus (Eksigent, Dublin, CA, USA) for ultra-high-pressure parallel LC with an additional loading pump for fast sample loading and washing. For this work, all LC systems were configured with $300 \mu \mathrm{m}$-i.d 5-mm PepMap C18 trap columns (Dionex, Sunnyvale, CA, USA), 15-cm $300 \mu$ m-i.d. ChromXP C18 columns supplied by Eksigent and running linear gradients, all from $4 \%$ to $44 \%$ acetonitrile in $0.05 \%$ formic acid, but of different lengths.

The FTICR is a solariX 12 T FTICR (Bruker Daltonics, Bremen, Germany) equipped with an Apollo II ESI source and external quadrupole for precursor ion selection and/or MS/MS outside the cell. In the FTICR ion trap cluster, this quadrupole is only used as an ion guide with transmission optimized for $m / z$ 400-1000, which includes most doubly- and triply charged tryptic peptides. Typically $2^{20}\left(\sim 10^{6}\right)$ data points are acquired per spectrum, and one spectrum is acquired every 2-3 s.

The ion traps in the particular instrument cluster used to generate all data shown here were of two models, both from Bruker Daltonics, with one HCT ultra PTM Discovery system for collision-induced dissociation (CID) and electron-transfer dissociation (ETD), and one standard HCT ultra system exclusively for CID combined in pairs and connected to a single LC system. After each MS scan, up to five abundant multiply charged species in $m / z$ 300-1300 were selected for MS/MS and excluded for $1 \mathrm{~min}$ after being selected twice. For spectral counting no active precursor exclusion was used.

Each mass spectrometer is controlled by a dedicated computer, but all instruments are monitored from a single desk with two monitors using dual 4-port KVM switches. The LC systems are controlled using the HyStar 3.2-3.4 with a plug-in from the LC manufacturer, the ion traps by esquireControl 6.2 and the FTICR by apexControl 3.0, all from the instrument manufacturer. The acquired data from each mass spectrometer is automatically transferred to a dedicated server and processed as described below.

\section{Data Analysis}

Automation of data analysis tasks is essential for the easy operation of the instrument cluster. All data is 
continuously copied over a gigabyte/s Ethernet connection to a dedicated data processing server. By using a convention with delimited LC mass spectrometer species and unique sample identifiers in the HyStar sample lists, and consequently the resulting filenames, the ion trap data can be automatically searched against a species-specific sequence database using a local installation of X!Tandem [13]. The identified peptides are then used to align each ion trap dataset with the corresponding FTICR dataset from the same sample in the "hybrid instrument emulation mode," as previously described [10]. Additionally and optionally, identified and aligned peptides can also be used to internally calibrate the FTICR mass spectra [14], generating a hybrid peak list with sub-ppm precursor mass measurement uncertainty. The hybrid peak lists are then automatically searched against the same database but with a narrow precursor (peptide) mass tolerance window. The data analysis so far is performed in the background without any user input. The processing scripts and all software used for the alignment of LC-MS and LCMS/MS datasets and for integrating peak areas in the LC-MS data will be freely available as open source on http://www.ms-utils.org/cluster. All analyses can also be performed in batch-mode and off-line, allowing the use of other search engines such as Mascot [15] or Phenyx [16] running on separate servers. For the data presented in this paper, we exclusively relied on Mascot, as it is the most common of the search engines available in our lab. All quantitative analyses are currently only performed in batch-mode. All FTICR datasets are searched for all identified peptides in a narrow retention time window and a very narrow $m / z$ window (typically $\pm 2 \mathrm{ppm}$, as low signal-to-noise peaks have larger mass measurement errors) to retrieve quantitative information of all identified peptides in each biological replicate. The quantitation is done by adding all signals in this narrow $\mathrm{m} / \mathrm{z}$ and time window. This only requires that the last chromatographic dimension used with the ion traps for identification can be aligned with that used with the FTICR for quantitation. All peptide intensities are then summed to total protein intensity.

\section{Results}

\section{FTICR-Ion Trap Cluster}

The basic working principle of the FTICR-ion trap cluster (Figure 1) is that each compound in each sample is analyzed twice, once on the FTICR for accurate mass determination and once on an ion trap for MS/MS. The high resolving power of the 12 tesla (T) FTICR mass spectrometer allows many simultaneous accurate mass determinations also in very complex spectra. All mass spectrometers in the cluster are coupled on-line to parallel ultra-high-pressure LC systems for efficient use of the mass spectrometers and high chromatographic peak capacity. The new and enabling idea behind the FTICR-ion trap cluster is the use of short and long chromatographic gradients, respectively, with a single accurate mass/high-resolution mass spectrometer, here a 12 T FTICR, and multiple rapid and sensitive MS/MS instruments, here $3 \times 2$ state-of-the-art ion traps. These two independent LC separations are performed in such a way that the single FTICR can keep up with the throughput of and provide accurate MS data to the MS/MS data from the multiple ion traps, for instance

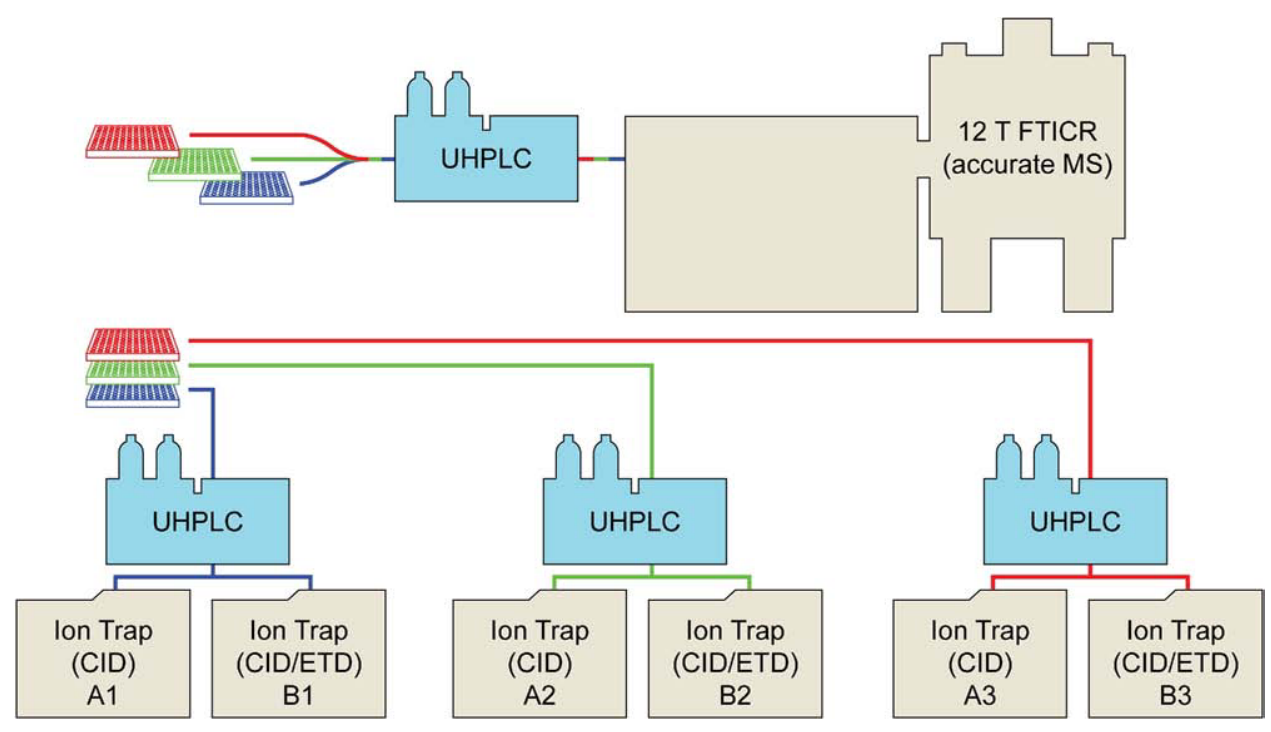

Figure 1. The FTICR-ion trap cluster consists of six ion traps grouped in three pairs. In each pair, the eluent from one capillary LC system is split in two, with half going to a CID-only ion trap and half to an ETD-capable ion trap. The ion traps are used for fast MS/MS acquisition. The FTICR component consists of an identical capillary LC system as used with the ion traps and a $12 \mathrm{~T}$ solariX Qq-FTICR. Although capable of MS/MS and even $\mathrm{MS}^{n}$, the FTICR is used exclusively for accurate MS and quantitation in this instrument cluster. 
by compressing the gradient a factor equal to the number of ion traps or ion trap "modules" in the system, where a module is defined as one or more ion traps coupled to a single LC (see Figure 1). The specific ion trap cluster described here combines one ion trap for ETD [17] with one ion trap for CID in each of three such modules in the system. The use of separate instruments allows different configurations, the most straightforward being one LC system per ion trap.

However, there are some advantages in the use of two ion traps in parallel to one LC. In these ion traps, there is significant dead time in the switching between CID and ETD. Using two ion traps where one is dedicated to CID and one to ETD not only improves duty cycles, but in principle allows the instruments to be tuned and optimized for a particular dissociation method, including precursor ion selection criteria. For complex samples, it is also well known that ion traps cannot sample all detected peptides for MS/MS on any reasonable chromatographic timescale. It is also feasible to combine data from split samples acquired on two or more LC-ion trap systems with one LC per ion trap. The data acquisitions then need not be concurrent, but the results from one acquisition can be used to make an exclusion list for the next.

Automated integration of accurate mass determinations from the FTICR with a large number of MS/MS spectra from the ion traps is key in the instrument cluster, and alignment and combination of accurate MS data from FTICR and MS/MS data from ion trap mass spectrometers improves confidence in peptide identifications and makes it possible to identify MS/MS spectra of lower quality, resulting in more peptide and protein identifications at a given false discovery rate [10]. The ion trap and FTICR data acquisitions are physically and timewise independent which allows the instrument cluster to be operated in different modes. We call two basic modes the "hybrid instrument emulation" mode and the "quantitative accurate mass and time tag" (QAMT) mode, respectively (Figure 2).

In the hybrid instrument emulation mode, each sample is analyzed on both the ion trap and FTICR platforms, and the data aligned and combined to hybrid datasets with accurate precursor ion masses from FTICR and MS/MS from two ion traps, for instance both CID or one CID and one ETD (a detailed comparison of different CID/ETD schemes was recently published by Leinenbach et al. [18]. These hybrid datasets are complete as each tandem mass spectrum is supplied with an accurate precursor mass, and similar in quality to what would be obtained from a hypothetical ETDcapable $12 \mathrm{~T}$ ion trap-FTICR instrument. If the FTICR dataset is acquired first, inclusion lists can be made based on the accurate mass for subsequent MS/MS in the ion traps, just as in a hybrid instrument. The latest version of the ion trap control software allows for scheduled precursor lists with $\mathrm{m} / \mathrm{z}$ as well as elution time windows of precursors to be selected for MS/MS. The QAMT mode uses a different set of samples, or fractions of a representative or pooled sample for identification of peptides quantified in individual biological replicates, time points or experimental conditions by the FTICR. This is similar to the AMT scheme by Smith et al. but the emphasis here is on the FTICR providing both quantitation and accurate MS to increase confidence in peptide identifications. The FTICR-ion trap cluster also allows the on-the-fly generation of the AMT database.

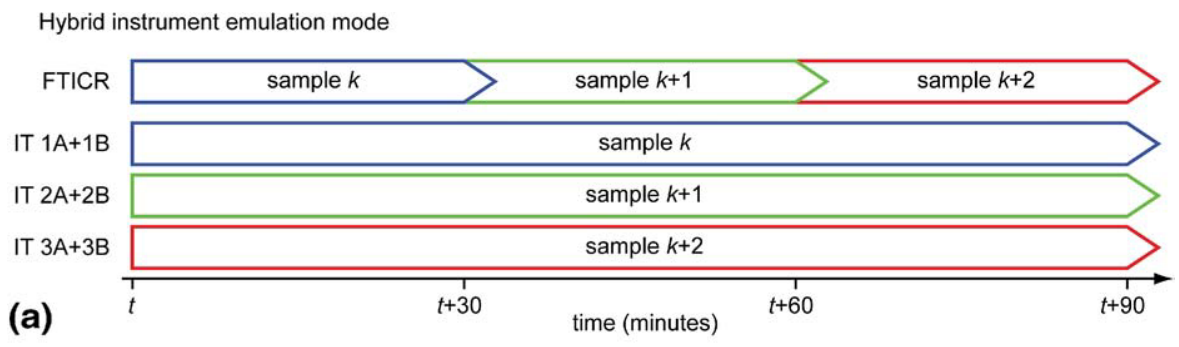

Quantitative AMT mode (one condition, time point or sample type)
(b)
(b)

Figure 2. Repeating scheduling blocks for the FTICR-ion trap cluster with $3 \times 2$ ion traps in the hybrid instrument emulation mode (a) and quantitative accurate mass and time mode (b). If these schedules are kept, the FTICR and ion trap cluster will require exactly the same time for the analysis of a batch of samples. It is not necessary to strictly adhere to such time schedules, but they are helpful in maximizing sample throughput and obtainable data quality. 


\section{Dataset Alignment and Chromatographic Compression}

An E. coli whole-cell lysate obtained as described in the Methods section was analyzed using linear chromatographic gradients of 9, 10, 11.3, 12.9, 15, 18, 22.5, 30, 45, and 90 min corresponding to compression ratios of 10:1 down to 1:1. The previously described alignment algorithm [10] had been designed to be as general and robust as possible, and was found to be insensitive to chromatographic time scales. Without modification, the algorithm correctly aligned datasets from the different chromatographic time scales (Figure 3 ). The elution times depend linearly $\left(R^{2}>0.99\right)$ on compression ratio, as expected (Figure 4a). However, the alignment is more robust, or reproducible, for lower compression ratios, as indicated by the larger variation in the slope of the piecewise linear alignment. This is caused by the software having to look for the precursor $\mathrm{m} / \mathrm{z}$ in a larger relative time window at higher compression ratios. The alignment is very robust up to at least a compression ratio of $3: 1$, which is the number of ion trap modules and the highest compression ratio in our setup.

Another cost of compression of chromatographic gradients is that fewer features (here peptides) are observed at higher compression ratios. This can be quantified as the fraction of species tentatively identified by MS/MS in the ion traps and above the detection

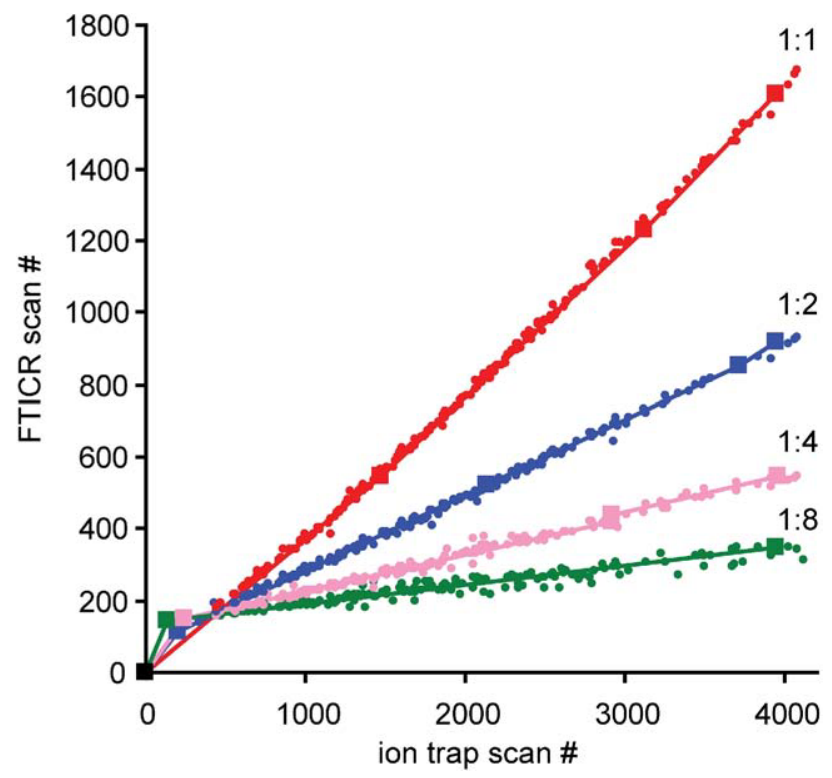

Figure 3. Automatic alignments (lines) of FTICR and ion trap datasets with chromatographic compression FTICR:ion trap 1:1, $1: 2,1: 4$, and $1: 8$, showing the peptide features contributing to the fitness function in the genetic algorithm used for alignment (dots). All alignments were performed allowing a 25 scan residual standard error, mass measurement error tolerance $\pm 1 \mathrm{ppm}$ and 262 unique peptides identified with a Mascot ion score cutoff 30. The squares represents the breakpoints in the piecewise linear alignments. No parameters needed to be adjusted to align chromatograms of different time scales.
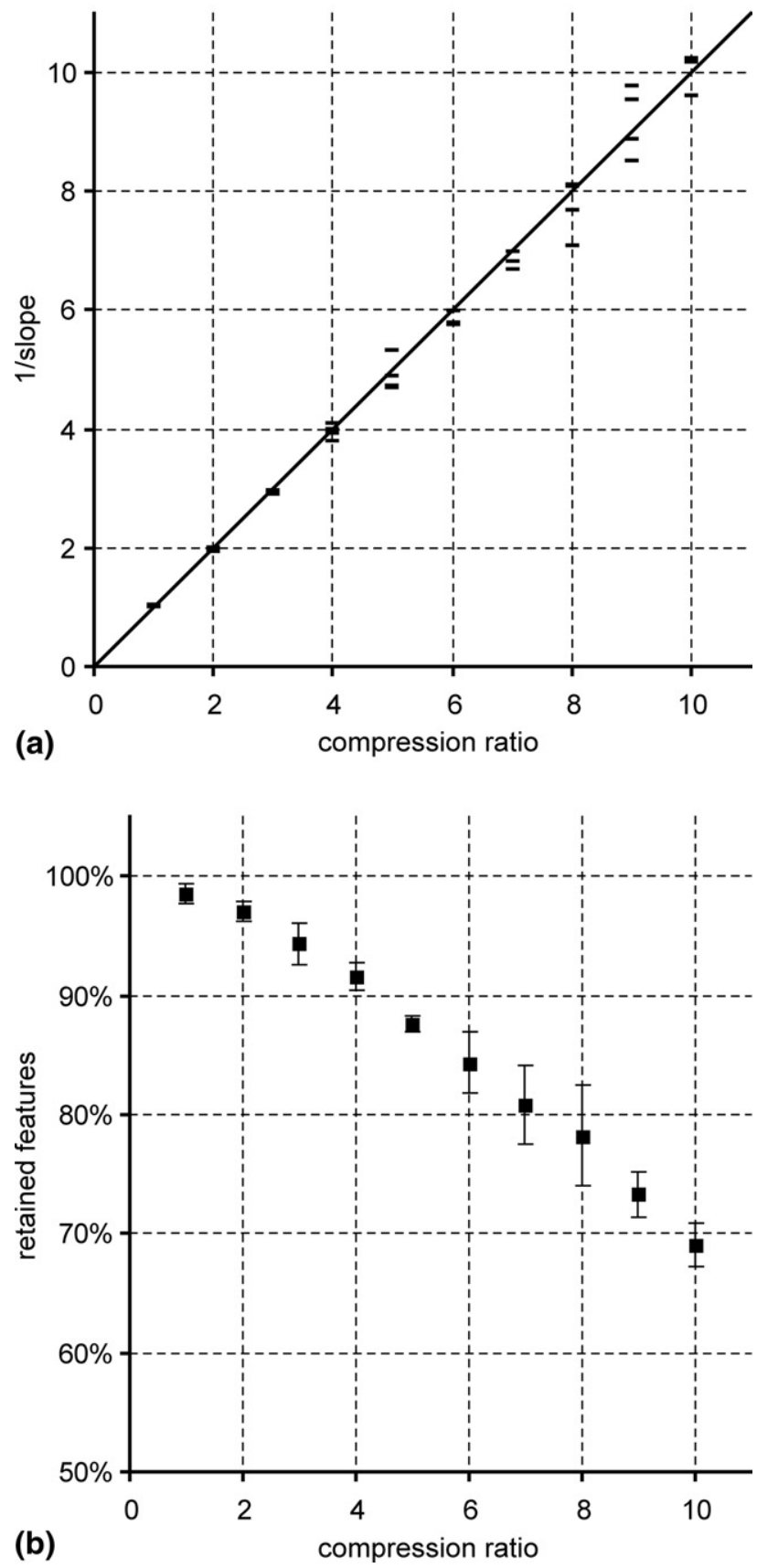

Figure 4. The slope of the main segment of the piecewise linear alignment as a function of chromatographic compression (a). The plotted slope for four replicates at each compression ration was normalized to compensate for the different data acquisition rates in the FTICR and ion traps. The retention times of matched peptide features have a linear dependence on the length of the chromatographic gradient, but alignment is more difficult at higher compression ratios. More features are also lost, or not observed, within $\pm 1 \mathrm{ppm}$ in the expected elution time window, the more the chromatographic gradient is compressed on the FTICR (b). These numbers are derived from the relatively abundant peptides identified by MS/MS. For lower abundant species not identified by MS/MS, the fraction lost is likely larger.

limit and within $\pm 1 \mathrm{ppm}$ of the theoretical mass in the FTICR. The fraction of identified peptides retained as a function of chromatographic compression is shown in 
Figure $4 \mathrm{~b}$. Note that the percentage of preserved features decreases smoothly as a function of chromatographic compression, from around $98 \%$ with no compression to just below $70 \%$ with compression from 90 to $9 \mathrm{~min}$. At 3-fold compression from 90 to $30 \mathrm{~min}, 94 \%$ of the peptides selected for MS/MS are retained. This limited loss is compensated with nearly $200 \%$ gain in throughput.

There will probably always be some tradeoff between coverage and measurement throughput in proteomics. The FTICR-ion trap cluster is no exception, even though it is designed to provide a relatively deep coverage using high-field FTICR with a high throughput using chromatographic compression and multiple ion traps. It is important to keep in mind that these numbers refer to peptides selected for MS/MS and producing good CID spectra in the ion traps, and not all features detectable by MS. The $2 \%$ "lost" peptides with identical chromatographic gradients is comparable to what would be expected between repeated analyses on the same system and are comprised of erroneous peptide identifications from the ion trap data alone, peptides measured outside the tolerated and searched $\mathrm{m} / \mathrm{z}$ window in the FTICR, and peptides falling below the detection limit in the FTICR. Gygi and coworkers have reported "losses" of a similar magnitude between LTQonly and LTQ-FT datasets, where slightly more MS/MS spectra are acquired and a few more (unmodified) proteins identified using only the LTQ rather than the hybrid LTQ-FT $[19,20]$, so this is not a phenomenon unique to the FTICR-ion trap cluster. In the FTICR-ion trap cluster, the MS/MS data is acquired in the ion traps completely independently from the FTICR, without any time loss.

\section{High Throughput Quantitative Proteomics}

Label-free quantitation using FTICRMS is more precise and covers a larger dynamic range in relative protein abundance than label-free quantitation using only ion traps, for instance with the emPAI spectral counting method [21] (Figure 5). Good agreement with calculated isotopic distributions and precise relative quantitation using ${ }^{15} \mathrm{~N}$-labeling and FTICRMS has also been reported previously [22]. The FTICR-ion trap cluster is ideally suited to study proteome dynamics, analyzing large cohorts of similar samples. We have chosen to illustrate the throughput and applicability of the instrument cluster with a time-course study of the glucoselactose diauxie [11] in E. coli. A subset of the data is shown in Figure 6 and compared with a recently published gene expression study [12]. The diauxie experiment serves as a positive control, as we expect to see $\beta$-galactosidase to be the most up-regulated protein during the glucose-lactose shift. A 10 -fold increase in abundance of this protein could also be observed in each of the three replicate time series (Figure 6). This quantity and quality of data can be routinely generated in less $24 \mathrm{~h}$ using the FTICR-ion trap cluster, illustrating

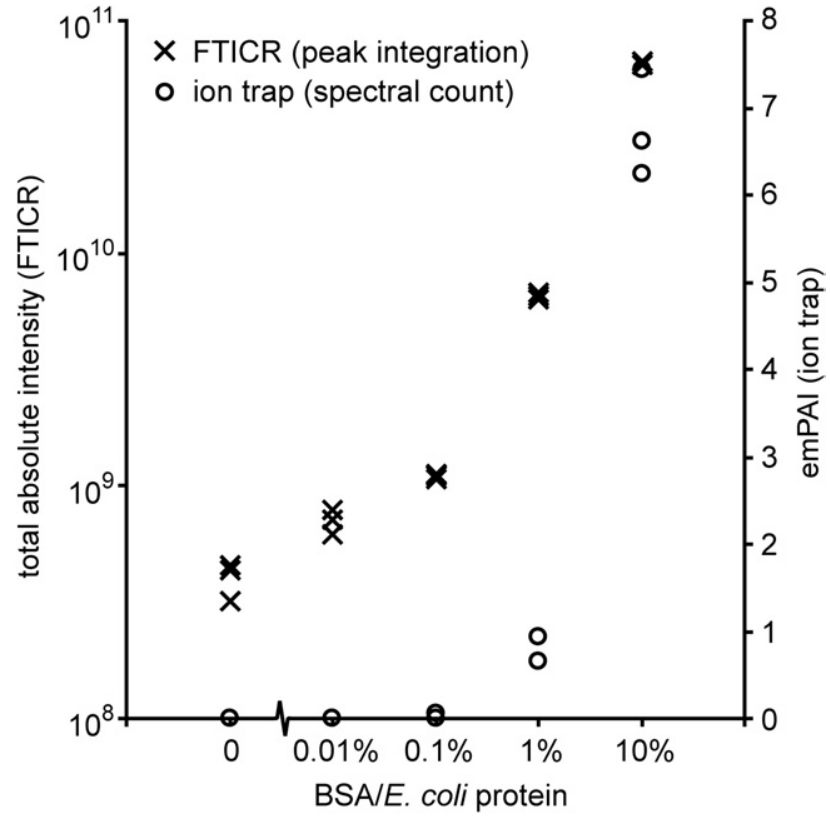

Figure 5. Comparison of technical reproducibility (quantitative precision) and dynamic range between FTICR and the emPAI [21] method for label-free quantitation using three replicate measurements of BSA spiked in a constant background of $E$. coli cell lysate at each of five relative abundance levels: $0,0.01 \%, 0.1 \%, 1$, and $10 \%$ of the background of E. coli protein. The FTICR signal abundance is the total peak intensity integrated (40 scans around the chromatographic maximum in a region $\pm 5 \mathrm{ppm}$ and \pm 25 scans from the $m / z$ and retention time predicted from the ion trap MS/MS data) over the chromatographic peaks of 28 identified BSA peptides, without normalization. No BSA peptides were identified in the ion trap at the 0 or $0.01 \%$ spike level, and only a single peptide in one of the replicates at the $0.1 \%$ level. At the $1 \%$ and $10 \%$ levels, spectral counting works well, with relative standard error of $19 \%$ at the $1 \%$ spike level and $9 \%$ at the $10 \%$ spike level. Conversely, the relative standard error of the FTICR measurement is around $3 \%$ at and above the $0.1 \%$ level, and $12 \%$ at the $0.01 \%$ level. Both the limit of detection and limit of quantitation, as functions of relative abundance, are approximately two orders of magnitude lower in the FTICR method than with spectral counting from the same sample. In this comparison, the same spiked samples and 90-min gradients were used for the ion trap and FTICR analyses. The figure shows data from two columns, one used with the FTICR and one with the ion trap.

the power of the system in large-scale proteomic studies.

With sufficient protein or peptide fractionation or enrichment, the number of identified peptide features may eventually exceed the number of observable peptide features in the FTICR data. The more identified peptides, the larger the risk of false matches between FTICR and ion trap data. However, the situation may be improved by good mass measurement accuracy $(<0.5$ ppm standard error in the 12 T FTICR after automatic internal calibration) chromatographic reproducibility and restricting the number of peptides of similar mass and elution times, which is essentially the idea behind the accurate mass and time tag approach. Most importantly, the false matches between the FTICR and ion trap data is expected to increase with the difference 

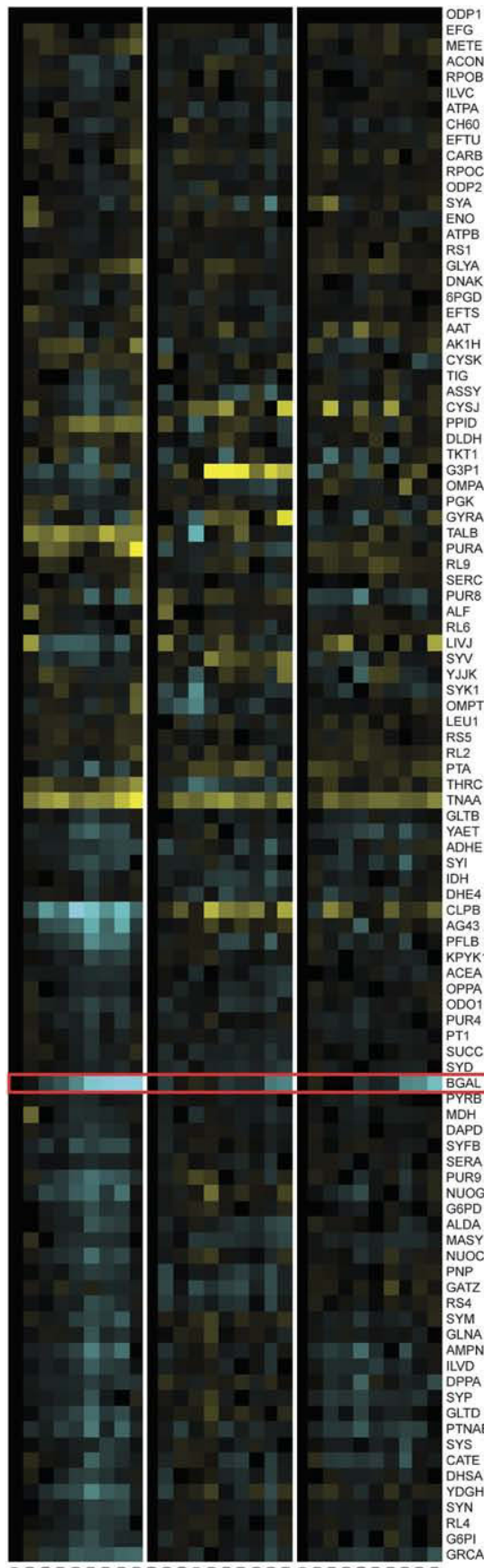

(a)

$$
\text { time ( } \mathrm{min})
$$

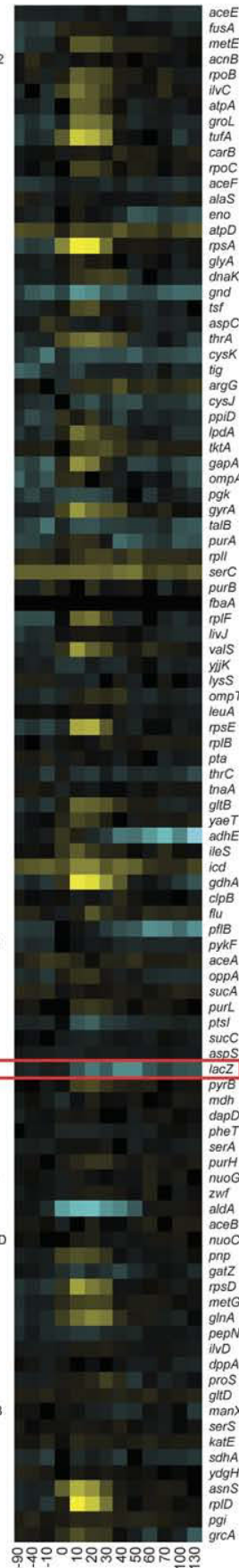

(b) time ( $\mathrm{min})$
Figure 6. Protein (a) and gene (b) expression in Escherichia coli K12 during glucose-lactose diauxie, showing 100 out of 948 proteins (630 with more than one peptide) quantified at 10 time points across three biological replicates-a total of $27,492(18,270$ with multiple peptides) quantitative protein abundance measurements by the FTICR-ion trap cluster. Cyan color denotes increase, yellow decrease, and black no change. The times are relative to the onset of diauxic shift as observed by OD600 measurement. These data can be obtained in less than $24 \mathrm{~h}$ using the instrument cluster. The figure shows $\sim 10 \%$ of the total proteomic dataset (see Supplemental Information, which can be found in the electronic version of this article) compared with the corresponding gene expression data from a similar experiment by Traxler et al. [12]. The protein with the strongest increase in abundance during the glucose-lactose diauxic shift is $\beta$-galactosidase/lac $Z$, as expected (marked in red). between the individual samples analyzed by the FTICR and the sample or samples used for fractionation and peptide identification. Quantifying samples from individual time points or experimental conditions using one or more pooled samples from the same experiment for identification is likely to reduce the risk of false matches. In the pipeline described here, we also strive to use all available information, which includes using at least all peptides with a sequence unique to a protein for protein identification and quantitation. This way, the false positive rate can be much lower on the protein level than on the peptide level, the confidence generally increasing the more peptides are available for identification and quantitation per protein.

\section{Discussion}

The first implementation of an FTICR-ion trap cluster described here was designed for robustness and optimal instrument performance using state-of-the-art ion trap and $12 \mathrm{~T}$ FTICR mass spectrometers, capillary ultra-high-performance liquid chromatography systems and standard ESI source, operating at $2 \mu \mathrm{L} / \mathrm{min}$. We have chosen a $12 \mathrm{~T}$ system as it provides highperformance at a reasonable cost. The chromatographic alignment is more robust the more features are used for alignment, i.e., the more information that is available for alignment. However, the time required for evaluation of the fitness function in the genetic algorithm is proportional to the number of features or peptides used for alignment. In our experience, it is a good practice to limit the number of features to at most a few thousand [23]. For peptides, this is easily done by raising the search engine score threshold. At the other end, the algorithm does not need more than 30-40 matched peptides distributed over the chromatographic separation to produce a good alignment [10].

Fragmentation by CID is the most commonly used method for MS/MS in general as well in the ion trap cluster. However, peptides with labile post-translational modifications such as phosphorylation or glycosylation often lose these before producing sequence-specific backbone fragments by CID, thus preventing the exact localization of the post-translational modification. The recently introduced ETD in linear and three-dimensional ion traps is therefore extremely useful for primary structure determination of peptides containing posttranslational modifications, and has been rapidly implemented and accepted in the field. Electron-transfer dissociation, like electron capture dissociation [24] in Fourier transform ion cyclotron resonance (FTICR) mass spectrometers, cleaves $\mathrm{N}-\mathrm{C} \alpha$ bonds of a peptide backbone more or less evenly and cleavages are less dependent on amino acid sequence than in CID. Often labile post-translational modifications are retained after ETD of the backbone, making it possible to localize the modified amino acid residue. Each ion trap module in the FTICR-ion trap cluster therefore contains one ion trap equipped for ETD. 
The resolving power and dynamic range of the FTICR is taken full advantage of for quantitative peptide and protein measurements. The system is ideal for large-scale quantitative proteomic studies, using labelfree quantitation or stable-isotope labeling methods such as SILAC [25] or multiplexed ${ }^{15} \mathrm{~N}$-labeling [22]. Despite its performance, the cluster has some limitations. For instance, it is not ideally suited for iTRAQ [26, 27] measurements, as these require MS/MS for the relative quantitation. The use of capillary rather than nanoflow LC reduces absolute sensitivity, but we have chosen this option as the chromatography systems and ESI sources require considerably less maintenance than in typical nanoflow systems. This robustness is essential in large-scale studies. Data-dependent precursor ion selection based on accurate mass in real time is also not possible in the cluster. However, it is possible to first perform the FTICR analysis and then use the accurate mass information to construct so-called scheduled precursor lists (inclusion criteria based on time as well as $\mathrm{m} / \mathrm{z}$ ) for one or more of the ion traps. For instance, species suspected to be phosphorylated based on accurate mass measurement [28] could be targeted for MS/MS using ETD, or a combination of CID and ETD. Moreover, the loose coupling of the instrumentation and nonconcurrent (or not necessarily concurrent) acquisition of MS and MS/MS data allows great freedom in constructing and exploring novel schemes for datadependent acquisition, as the analyses of the MS data dos not have to be performed in real-time and integrated into the instrument control software.

To appreciate the QAMT analysis mode, one can consider the following experiment. Assume $n$ replicates of $b$ biological samples or experimental conditions are collected, in total $n \times b$ samples to be analyzed on a cluster with $c$ ion trap modules. Each sample and replicate is digested by trypsin and analyzed by LCFTICR using reversed-phase chromatography only. In parallel, the proteins or digests of the replicates of each type of biological sample are pooled and fractionated by, for instance, SDS-PAGE (proteins) or strong cation exchange chromatography (peptides), into $c \times n$ fractions, for a total of $c \times n \times b$ fractions. For three ion trap modules and four biological replicates, 12 fractions would be collected for each of the $b$ types of biological sample. These fractions are subsequently analyzed on one of the ion trap modules in the cluster. In such a case, the same length chromatographic gradients can be used with the FTICR and the ion traps, with the total analysis time on the FTICR exactly matching that of the ion traps. For example, with 60-min LC methods and three ion trap modules, the independent quantitative analysis of four biological replicates of six different conditions or time points with 12 SCX peptide fractions collected for each takes $24 \mathrm{~h}$, generating 24 LC-FTICR analyses for quantitation, and 72 ion trap LC-MS/MS datasets for identification. The peptides are quantified in a similar manner as in the AMT protocol [6] by integrating the area under the LC-MS peak in the FTICR data for the major peak in a narrow $m / z$ range and the predicted retention time window. All "AMT tags" are confirmed by MS/MS on one of the ion traps on at least one similar sample from the same study, although not necessarily for every individual sample, treatment, or time point. This also means that peptides (and consequently, proteins) can be quantified at much lower levels than are needed for confident identification by MS/MS, which is also illustrated by the BSA measurements summarized in Figure 5. Under ideal LC conditions, the elution time of a peptide in the last, reversedphase dimension does not depend on which protein or peptide separation or separations were used in the prior dimensions, e.g., which SCX fraction is analyzed, and the elution times in the last (reversed-phase) dimension on the LC-ion trap systems can be aligned with those in the only (reversed-phase) LC separation with the FTICR. This QAMT scheme is feasible as the 12 T FTICR has sufficient resolving power and dynamic range for the $c \times n$-fold higher sample complexity compared to the ion trap LC-MS/MS. Analogous schemes can be constructed for any number and type of peptide or protein fractionation before the final reversed-phase separation, for instance, proteins can be fractionated by SDS-PAGE and digested for identification by LCMS/MS while individual samples are analyzed by LC-FTICR MS only.

As illustrated by the E. coli diauxie example, the QAMT mode is particularly useful for comparison of relatively similar samples, such as a series incorporating different time points, treatments, or experimental replicates. It is then feasible to use a two-dimensional separation before MS/MS, where the second dimension is of the same type, e.g., reversed-phase, as the one used as the only LC dimension for FTICR-MS. A high-field FTICR mass spectrometer is capable to resolve and detect more than 10,000 peptides in a relative short chromatographic separation, and the additional dimension of separation aids the identification in the ion traps. Complex peptide mixtures have even been analyzed by direct infusion and high-field FTICR, i.e., without prior separation [29, 30]. For instance, we recently demonstrated that even by direct infusion, it is possible to detect and resolve most peptides in a combinatorial library with more than 1000 unique elemental compositions spanning a factor 36 in concentration [31]. For a very large number of biological replicates, the scheme becomes similar to the AMT tag protocol developed by Smith et al. [7, 32, 33], where the "AMT tags" are verified at least once by MS/MS, placed in a database, and aligned through normalization of the retention times with the accurate mass data from an FTICR [9]. A particular advantage of the AMT or QAMT mode is that it is still possible to quantify peptides and proteins in samples where they are present at a lower concentration than would be required to produce good MS/MS data, which is required to generate any quantitative information in many other methods such as iTRAQ or spectral counting [21, 34]. 
The FTICR-ion trap cluster provides quantitative proteomics data of a similar quality with comparable throughput to that of multiple hybrid ion trap-FTICR or ion trap-Orbitrap instruments at lower cost and infrastructure requirements. The instrument cluster has a few limitations, but in turn opens up additional possibilities for data-dependent MS/MS acquisition, and can serve as a test bed for the design and development of hybrid instruments with a single accurate mass analyzer and multiple ion traps for MS/MS. The cluster design and idea of chromatographic compression between LC-MS and LC-MS/MS is not limited to the use of a high-field FTICR and six ion traps, but may also be applicable to the combination of one MS-only TOF or Orbitrap with one or more MS/MS-capable mass spectrometers, albeit with lower mass accuracy and resolving power than any high-field FTICR instrument. The cluster scheme provides an inexpensive means to adding accurate mass capability in laboratories already operating one or more ion trap instruments for LC-MS / MS. All components in the cluster are individually exchangeable, which provides a high degree of flexibility that can be used to continuously upgrade the system. For instance, during the first year of operation, the 12 T FTICR front-end was upgraded from a previous apex ultra model to the recently introduced solariX.

\section{Acknowledgments}

The authors thank Bart Schoenmaker, Rico Derks, Hannah Scott, and René van Zeijl for technical assistance, and Paul Hensbergen for helpful comments on the manuscript.

\section{Appendix A Supplementary Material}

Supplementary material associated with this article may be found in the online version at doi:10.1016/ j.jasms.2010.02.001.

\section{References}

1. March, R. E. An Introduction to Quadrupole Ion Trap Mass Spectrometry. J. Mass Spectrom. 1997, 32, 351-369.

2. Morris, H. R.; Paxton, T.; Dell, A.; Langhorne, J.; Berg, M.; Bordoli, R. S.; Hoyes, J.; Bateman, R. H. High Sensitivity Collisionally-Activated Decomposition Tandem Mass Spectrometry on a Novel Quadrupole/ Orthogonal-Acceleration Time-of-Flight Mass Spectrometer. Rapid Commun. Mass Spectrom. 1996, 10, 889-896.

3. Makarov, A.; Denisov, E.; Kholomeev, A.; Balschun, W.; Lange, O.; Strupat, K.; Horning, S. Performance Evaluation of a Hybrid Linear Ion Trap/Orbitrap Mass Spectrometer. Anal. Chem. 2006, 78, 2113-2120.

4. Syka, J. E.; Marto, J. A.; Bai, D. L.; Horning, S.; Senko, M. W.; Schwartz, J. C.; Ueberheide, B.; Garcia, B.; Busby, S.; Muratore, T.; Shabanowitz, J.; Hunt, D. F. Novel Linear Quadrupole Ion Trap/FT Mass Spectrometer: Performance Characterization and Use in the Comparative Analysis of Histone H3 Post-Translational Modifications. J. Proteome Res. 2004, 3, 621-626.

5. O'Connor, P.; B.; Pittman, J. L.; Thomson, B. A.; Budnik, B. A.; Cournoyer, J. C.; Jebanathirajah, J.; Lin, C.; Moyer, S.; Zhao, C. A New Hybrid Electrospray Fourier Transform Mass Spectrometer: Design and Performance Characteristics. Rapid Commun. Mass Spectrom. 2006, 20, 259-266.

6. Pasa-Tolic, L.; Masselon, C.; Barry, R. C.; Shen, Y.; Smith, R. D. Proteomic Analyses Using an Accurate Mass and Time Tag Strategy. Biotechniques 2004, 37, 621-624, 626-633, 636 passim.

7. Petritis, K.; Kangas, L. J.; Ferguson, P. L.; Anderson, G. A.; Pasa-Tolic, L.; Lipton, M. S.; Auberry, K. J.; Strittmatter, E. F.; Shen, Y.; Zhao, R.; Smith,
R. D. Use of Artificial Neural Networks for the Accurate Prediction of Peptide Liquid Chromatography Elution Times in Proteome Analyses. Anal. Chem. 2003, 75, 1039-1048.

8. Petritis, K.; Kangas, L. J.; Yan, B.; Monroe, M. E.; Strittmatter, E. F.; Qian, W. J.; Adkins, J. N.; Moore, R. J.; Xu, Y.; Lipton, M. S.; Camp, D. G. II Smith, R. D. Improved Peptide Elution Time Prediction for ReversedPhase Liquid Chromatography-MS by Incorporating Peptide Sequence Information. Anal. Chem. 2006, 78, 5026-5039.

9. Jaitly, N.; Monroe, M. E.; Petyuk, V. A.; Clauss, T. R.; Adkins, J. N.; Smith R. D. Robust Algorithm for Alignment of Liquid Chromatography-Mass Spectrometry Analyses in an Accurate Mass and Time Tag Data Analysis Pipeline. Anal. Chem. 2006, 78, 7397-7409.

10. Palmblad, M.; Mills, D. J.; Bindschedler, L. V.; Cramer, R. Chromatographic Alignment of LC-MS and LC-MS/MS Datasets by Genetic Algorithm Feature Extraction. J. Am. Soc. Mass Spectrom. 2007, 18, 1835-1843.

11. Jacob, F.; Monod, J. Genetic Regulatory Mechanisms in the Synthesis of Proteins. J. Mol. Biol. 1961, 3, 318-356.

12. Traxler, M. F.; Chang, D. E.; Conway, T. Guanosine 3',5'-Bispyrophosphate Coordinates Global Gene Expression During Glucose-Lactose $\mathrm{Di}$ auxie in Escherichia coli. Proc. Natl. Acad. Sci. U.S.A. 2006, 103, 2374-2379.

13. Craig, R.; Beavis, R. C. TANDEM: Matching Proteins with Tandem Mass Spectra. Bioinformatics. 2004, 20, 1466-1467.

14. Palmblad, M.; Bindschedler, L. V.; Gibson, T. M.; Cramer, R. Automatic Internal Calibration in Liquid Chromatography/Fourier Transform Ion Cyclotron Resonance Mass Spectrometry of Protein Digests. Rapid Commun. Mass Spectrom. 2006, 20, 3076-3080.

15. www.matrixscience.com.

16. Colinge, J.; Masselot, A.; Giron, M.; Dessingy, T.; Magnin, J. OLAV: Towards High-Throughput Tandem Mass Spectrometry Data Identification. Proteomics 2003, 3, 1454-1463.

17. Syka, J. E.; Coon, J. J.; Schroeder, M. J.; Shabanowitz, J.; Hunt, D. F. Peptide and Protein Sequence Analysis by Electron Transfer Dissociation Mass Spectrometry. Proc. Natl. Acad. Sci. U.S.A. 2004, 101, $9528-$ 9533.

18. Leinenbach, A.; Hartmer, R.; Lubeck, M.; Kneissl, B.; Elnakady, Y. A.; Baessmann, C.; Muller, R.; Huber, C. G. Proteome Analysis of Sorangium cellulosum employing 2D-HPLC-MS/MS and Improved Database Searching Strategies for CID and ETD Fragment Spectra. J. Proteome Res. 2009, 8, 4350-4361

19. Haas, W.; Faherty, B. K.; Gerber, S. A.; Elias, J. E.; Beausoleil, S. A.; Bakalarski, C. E.; Li, X.; Villen, J.; Gygi, S. P. Optimization and Use of Peptide Mass Measurement Accuracy in Shotgun Proteomics. Mol. Cell. Proteom. 2006, 5, 1326-1337.

20. Bakalarski, C. E.; Haas, W.; Dephoure, N. E.; Gygi, S. P. The Effects of Mass Accuracy, Data Acquisition Speed, and Search Algorithm Choice on Peptide Identification Rates in Phosphoproteomics. Anal. Bioanal. Chem. 2007, 389, 1409-1419.

21. Ishihama, Y.; Oda, Y.; Tabata, T.; Sato, T.; Nagasu, T.; Rappsilber, J.; Mann, M. Exponentially Modified Protein Abundance Index (emPAI) for Estimation of Absolute Protein Amount in Proteomics by the Number of Sequenced Peptides Per Protein. Mol. Cell. Proteom. 2005, 4 1265-1272.

22. Palmblad, M.; Mills, D. J.; Bindschedler, L. V. Heat-Shock Response in Arabidopsis thaliana Explored by Multiplexed Quantitative Proteomics Using Differential Metabolic Labeling. J. Proteome Res. 2008, 7, 780-785.

23. Nevedomskaya, E.; Derks, R.; Deelder, A. M.; Mayboroda, O. A.; Palmblad, M. Alignment of Capillary Electrophoresis-Mass Spectrometry Datasets Using Accurate Mass Information. Anal. Bioanal. Chem. 2009, 395, 2527-2533.

24. Zubarev, R. A.; Kelleher, N. L.; McLafferty, F. W. Electron Capture Dissociation of Multiply Charged Protein Cations. A Nonergodic Process. J. Am. Chem. Soc. 1998, 120, 3265-3266.

25. Ong, S. E.; Blagoev, B.; Kratchmarova, I.; Kristensen, D. B.; Steen, H. Pandey, A.; Mann, M. Stable Isotope Labeling by Amino Acids in Cell Culture, SILAC, as a Simple and Accurate Approach to Expression Proteomics. Mol. Cell. Proteom. 2002, 1, 376-386.

26. Ross, P.; Dey, S.; Pillai, S.; Daniels, S.; Williamson, S.; Guertin, S.; Minkoff, M.; X., C.; Purkayastha, B.; Pappin, D. Proceedings of the 54th ASMS Conference on Mass Spectrometry; Seattle, WA, May 28-June 1, 2006.

27. Ross, P. L.; Huang, Y. N.; Marchese, J. N.; Williamson, B.; Parker, K.; Hattan, S.; Khainovski, N.; Pillai, S.; Dey, S.; Daniels, S.; Purkayastha, S.; Juhasz, P.; Martin, S.; Bartlet-Jones, M.; He, F.; Jacobson, A.; Pappin, D. J. Multiplexed Protein Quantitation in Saccharomyces cerevisiae Using Amine-Reactive Isobaric Tagging Reagents. Mol. Cell. Proteom. 2004, 3, 1154-1169.

28. Spengler, B.; Hester, A. Mass-Based Classification (MBC) of Peptides: Highly Accurate Precursor Ion Mass Values Can Be Used to Directly Recognize Peptide Phosphorylation. J. Am. Soc. Mass Spectrom. 2008, 19, $1808-1812$

29. Horn, D. M.; Zubarev, R. A.; McLafferty, F. W. Automated Reduction and Interpretation of High Resolution Electrospray Mass Spectra of Large Molecules. J. Am. Soc. Mass Spectrom. 2000, 11, 320-332.

30. Palmblad, M.; Wetterhall, M.; Markides, K.; Håkansson, P.; Bergquist, J. Analysis of Enzymatically Digested Proteins and Protein Mixtures Using a 9.4 Tesla Fourier Transform Ion Cyclotron Mass Spectrometer. Rapid Commun. Mass Spectrom. 2000, 14, 1029-1034. 
31. Palmblad, M.; Drijfhout, J. W.; Deelder, A. M. High Resolution Mass Spectrometry for Rapid Characterization of Combinatorial Peptide Libraries. J. Comb. Chem. 2010, 12, 65-68.

32. Strittmatter, E. F.; Ferguson, P. L.; Tang, K.; Smith, R. D. Proteome Analyses Using Accurate Mass and Elution Time Peptide Tags with Capillary LC Time-of-Flight Mass Spectrometry. J. Am. Soc. Mass Spectrom. 2003, 14, 980-991.
33. Zimmer, J. S.; Monroe, M. E.; Qian, W. J.; Smith, R. D. Advances in Proteomics Data Analysis and Display Using an Accurate Mass and Time Tag Approach. Mass Spectrom. Rev. 2006, 25, 450-482.

34. Old, W. M.; Meyer-Arendt, K.; Aveline-Wolf, L.; Pierce, K. G.; Mendoza, A.; Sevinsky, J. R.; Resing, K. A.; Ahn, N. G. Comparison of Label-Free Methods for Quantifying Human Proteins by Shotgun Proteomics. Mol. Cell. Proteom. 2005, 4, 1487-1502. 